\title{
A generalisation of a result of de la Vallée Poussin
}

\author{
Friedrich Pillichshammer*
}

Friedrich Pillichshammer promovierte im Jahr 1999 im Fach Mathematik an der Universität Salzburg. Seit 2003 ist er außerordentlicher Professor an der Johannes Kepler Universität in Linz.

\section{Introduction}

If $A=\{a n+b: n \in \mathbb{N}\}$ with $a \in \mathbb{N}$ and $0 \leq b<a$, then a century-old result from de la Vallée Poussin [10] states that for all $x \geq 1$ we have

$$
\sum_{d \leq x} 1_{A}(d)\left\{\frac{x}{d}\right\}=\frac{x}{a}(1-\gamma)+O(\sqrt{x}),
$$

where $\gamma$ is Euler's constant, $\gamma:=\lim _{y \rightarrow \infty}\left(\sum_{n \leq y} 1 / n-\log y\right)$, where $1_{A}(t)$ is the characteristic function of the set $A \subseteq \mathbb{N}$, i.e., $1_{A}(t)=1$ if $t \in A$ and 0 otherwise, $\{t\}=t-\lfloor t\rfloor$ and $\sum_{d \leq x}$ means summation over all integers $d$ such that $1 \leq d \leq x$. A proof of this result

\footnotetext{
* The author is supported by the Austrian Science Foundation (FWF), Project S9609, that is part of the Austrian National Research Network "Analytic Combinatorics and Probabilistic Number Theory".
}

Die Euler-Mascheroni Konstante $\gamma$ ist definiert als der Grenzwert der Differenz zwischen der harmonischen Partialsumme $\sum_{n \leq x} 1 / n$ und $\log x$, wenn $x$ gegen unendlich strebt. Wie e und $\pi$ tritt $\gamma$ in verschiedensten Teilgebieten der Mathematik auf. Im Jahr 1898 veröffentlichte Charles de la Vallée Poussin einen Artikel in dem er u.a. folgendes zeigte: Dividiert man eine natürliche Zahl $n$ der Reihe nach durch alle Zahlen $d=1, \ldots, n$ dann strebt für beliebig wachsendes $n$ das Mittel der Bruchteile $\{n / d\}$ aller entstehenden Brüche gegen $1-\gamma$. Eine analoge Aussage ist richtig, wenn man lediglich durch alle Primzahlen zwischen 1 und $n$ dividiert. In diesem Beitrag befasst sich der Autor mit Verallgemeinerungen dieser Resultate, indem er einerseits den Bereich der Divisoren $d$ einschränkt und andererseits die Bruchteile durch eine Funktion $f(d)$ gewichtet. Dabei lassen sich in vielen Fällen analoge Formeln zum Resultat von de la Vallée Poussin angeben. 
can also be found in [7]. In [9] the error term $O(\sqrt{x})$ in (1) is improved to $O\left(x^{1 / 3} \log x\right)$. We remark that for $A=\mathbb{N}$ the result (1) is equivalent to Dirichlet's divisor problem. From (1) it follows that

$$
\sum_{d \leq x} 1_{A}(d)\left\{\frac{x}{d}\right\} \sim(1-\gamma) \sum_{d \leq x} 1_{A}(d) \text { as } x \rightarrow \infty .
$$

(As usual, $a(x) \sim b(x)$ as $x \rightarrow \infty$ means that $a(x) / b(x) \rightarrow 1$ as $x \rightarrow \infty$.)

The same asymptotic relation is true if $A$ consists of all prime numbers or of all prime powers. I.e., (2) remains valid if $A=\mathbb{P}$, see [10], or if $A=\left\{p^{\alpha}: p \in \mathbb{P}, \alpha \in \mathbb{N}\right\}$, see [7]. If $A=\left\{n^{r}: n \in \mathbb{N}\right\}$ for some fixed $r \in \mathbb{N}, r>1$, then it has been shown in [7], that

$$
\sum_{d \leq x} 1_{A}(d)\left\{\frac{x}{d}\right\} \sim\left(\frac{1}{1-r}-\zeta\left(\frac{1}{r}\right)\right) \sum_{d \leq x} 1_{A}(d) \text { as } x \rightarrow \infty .
$$

Here and throughout the paper $\zeta(\alpha)$ denotes the Riemann zeta function which is defined by $\zeta(\alpha):=\sum_{n=1}^{\infty} 1 / n^{\alpha}$ if $\alpha>1$ and by $\zeta(\alpha):=\lim _{x \rightarrow \infty}\left(\sum_{n \leq x} 1 / n^{\alpha}-x^{1-\alpha} /(1-\alpha)\right)$ if $0<\alpha<1$.

It is the object of this paper to show that (3) remains valid if $A=\{g(n): n \in \mathbb{N}\}$ where $g(n)$ is a polynomial of degree $r$ with coefficients in $\mathbb{N}_{0}$; see Theorem 1 in Section 2.

Motivated from these findings it is natural to investigate the asymptotic behaviour of the more general weighted fractional part sum

$$
\sum_{d \leq x} f(d)\left\{\frac{x}{d}\right\} \quad \text { as } \quad x \rightarrow \infty
$$

where $f(t)$ is an arithmetic function $f: \mathbb{N} \rightarrow \mathbb{R}_{0}^{+}$or any function $f:[1, \infty) \rightarrow \mathbb{R}_{0}^{+}$.

The most investigated case in literature is the case where $f(t)=t^{\alpha}$; see, for example, $[4,5,6]$. For instance it has been shown by Mercier [4] (in a bit more general setting) that for $\alpha>-1$ we have

$$
\sum_{d \leq x} d^{\alpha}\left\{\frac{x}{d}\right\}=c_{\alpha} x^{\alpha+1}+O\left(x^{\alpha+1 / 2}\right)
$$

where

$$
c_{\alpha}:=\int_{1}^{\infty} \frac{\{t\}}{t^{2+\alpha}} \mathrm{d} t= \begin{cases}\frac{1}{\alpha}-\frac{\zeta(\alpha+1)}{\alpha+1} & \text { if } \quad \alpha>-1, \alpha \neq 0, \\ 1-\gamma & \text { if } \alpha=0 .\end{cases}
$$

This result shows that for $\alpha>-1$ we have

$$
\sum_{d \leq x} d^{\alpha}\left\{\frac{x}{d}\right\} \sim C_{\alpha} \sum_{d \leq x} d^{\alpha} \text { as } x \rightarrow \infty,
$$

where

$$
C_{\alpha}:=(\alpha+1) c_{\alpha}= \begin{cases}\frac{\alpha+1}{\alpha}-\zeta(\alpha+1) & \text { if } \alpha>-1, \alpha \neq 0 \\ 1-\gamma & \text { if } \alpha=0\end{cases}
$$


We will show in Theorem 2 in Section 2 that an asymptotic relation of the form (6) holds if and only if $\alpha \geq-1$.

The paper is organised as follows: The principal results are stated in Section 2. In Section 3 we present some further examples and state an open problem. The proofs of the results will be given in Section 4 .

\section{The results}

First we provide an asymptotic formula for $\sum_{d \leq x} 1_{A}(d)\{x / d\}$ where $A=\{g(n): n \in \mathbb{N}\}$ and where $g(t)$ is a differentiable, increasing function such that $g(\mathbb{N}) \subseteq \mathbb{N}$.

Proposition 1 Let $g:[1, \infty) \rightarrow[1, \infty)$ be a differentiable, increasing function such that $g(\mathbb{N}) \subseteq \mathbb{N}$ and let $A=\{g(n): n \in \mathbb{N}\}$, then for any $x \geq g(1)$ we have

$$
\sum_{d \leq x} 1_{A}(d)\left\{\frac{x}{d}\right\}=\int_{1}^{x}\left\{\frac{x}{t}\right\}\left(g^{-1}\right)^{\prime}(t) \mathrm{d} t+O(y(x)),
$$

where $y(x)=y \in[1, x]$ is the unique solution of $y=g^{-1}(x / y)$.

If we restrict on polynomial functions $g$, then we can generalise the result of de la Vallee Poussin (2) and the asymptotic formula (3).

Theorem 1 Assume that $g(t)$ is a polynomial of degree $r \in \mathbb{N}$ with coefficients in $\mathbb{N}_{0}$. Let $A=\{g(n): n \in \mathbb{N}\}$, then we have

$$
\sum_{d \leq x} 1_{A}(d)\left\{\frac{x}{d}\right\} \sim D_{r} \sum_{d \leq x} 1_{A}(d) \quad \text { as } x \rightarrow \infty,
$$

where

$$
D_{r}= \begin{cases}1-\gamma & \text { if } r=1 \\ \frac{1}{1-r}-\zeta\left(\frac{1}{r}\right) & \text { if } r>1\end{cases}
$$

Now we turn to the case where $f(t)=t^{\alpha}$. We present a formula in the vein of Mercier's result (4) also for $\alpha=-1$. Although it is not the main object of this paper, our proof technique also allows an improvement of the error term in (4) for $\alpha \geq 0$.

Proposition 2 For $x \geq 1$ we have:

(i) If $\alpha>-1$, then $\sum_{d \leq x} d^{\alpha}\{x / d\}=c_{\alpha} x^{\alpha+1}+O\left(h_{\alpha}(x)\right)$, where

$$
h_{\alpha}(x)= \begin{cases}x^{\alpha} & \text { if } \alpha>1, \\ x(\log x)^{2 / 3} & \text { if } \alpha=1, \\ x^{(\alpha+1) / 2} & \text { if }-1<\alpha<1,\end{cases}
$$

and where $c_{\alpha}$ is as in (5).

(ii) $\sum_{d \leq x} d^{-1}\{x / d\}=\frac{1}{2} \log x+O\left((\log x)^{2 / 3}\right)$. 
Theorem 2 We have $\sum_{d \leq x} d^{\alpha}\{x / d\} \sim \widetilde{c}_{\alpha} \sum_{d \leq x} d^{\alpha}$ as $x \rightarrow \infty$ for some $\widetilde{c}_{\alpha} \geq 0$ if and only if $\alpha \geq-1$.

In case of existence of the asymptotic relation we have

$$
\widetilde{c}_{\alpha}= \begin{cases}1+\frac{1}{\alpha}-\zeta(\alpha+1) & \text { if } \alpha>-1, \alpha \neq 0 \\ 1-\gamma & \text { if } \alpha=0 \\ 1 / 2 & \text { if } \alpha=-1 .\end{cases}
$$

From Proposition 2 (or from (4)) it follows immediately that if $f(t)$ is a polynomial over $\mathbb{R}$ of degree $\operatorname{deg}(f)=\alpha$, then $\sum_{d \leq x} f(d)\{x / d\} \sim c_{\alpha} \sum_{d \leq x} f(d)$ where $c_{\alpha}$ is as in (5).

\section{Further results and open problem}

All of our results are of the form

$$
\sum_{d \leq x} f(d)\left\{\frac{x}{d}\right\} \sim c_{f} \sum_{d \leq x} f(d) \quad \text { as } x \rightarrow \infty
$$

with some proportionality constant $c_{f}>0$ depending only on $f$.

It is easy to show some further results in this vein as, for example:

(i) $\sum_{d \leq x}(\log d)\{x / d\} \sim(1-\gamma) \sum_{d \leq x} \log d$ as $x \rightarrow \infty$;

(ii) $\sum_{d \leq x} \Lambda(d)\{x / d\} \sim(1-\gamma) \sum_{d \leq x} \Lambda(d)$ as $x \rightarrow \infty$, where $\Lambda$ denotes the von Mangoldt function $\Lambda(d):=\log p$ if $d=p^{v}$ with $p \in \mathbb{P}$ and $v \in \mathbb{N}$ and 0 otherwise;

(iii) $\sum_{d \leq x} \sigma_{0}(d)\{x / d\} \sim(1-\gamma) \sum_{d \leq x} \sigma_{0}(d)$ as $x \rightarrow \infty$, where $\sigma_{0}(d):=\sum_{m \mid d} 1$;

(iv) $\sum_{d \leq x} \varphi(d)\{x / d\} \sim(2-\zeta(2)) \sum_{d \leq x} \varphi(d)$ as $x \rightarrow \infty$, where $\varphi$ is Euler's totient function.

Motivated by the given results and examples we state the following open problem:

Characterise the functions $f(t)$ for which an asymptotic equivalence of the form (8) holds.

It can be shown that a necessary condition for (8) to hold for some $f(t)$ is that $\sum_{d=1}^{\infty} f(d)=\infty$. On the other hand this condition is not a sufficient one. For example consider the function $f(t)=1_{A}(t)$ where $A=\left\{b^{n}: n \in \mathbb{N}_{0}\right\}$ for some integer $b \geq 2$. Then

$$
\sum_{d \leq x} f(d)\left\{\frac{x}{d}\right\}=\sum_{k=0}^{\left.\log _{b} x\right\rfloor}\left\{\frac{x}{b^{k}}\right\}= \begin{cases}0 & \text { if } x=b^{n} \\ n-\frac{1}{b-1}\left(b-b^{-n+1}\right) & \text { if } x=b^{n}-1\end{cases}
$$

Hence an asymptotic relation of the form (8) does not exist although $\sum_{d=1}^{\infty} f(d)=\infty$. If $f(t)$ grows very rapidly in the sense that for any $\varepsilon>0$ we have $\sum_{(1-\varepsilon) x<d \leq x} f(d) \sim$ $\sum_{d \leq x} f(d)$ as $x \rightarrow \infty$, then one can prove that $\sum_{d \leq x} f(d)\{x / d\}=o\left(\sum_{d \leq x} f(d)\right)$ as $x \rightarrow \infty$. Functions satisfying this growing condition are, for example, $f(\bar{d})=d$ !, $f(d)=\exp (d)$ or, more general, $f(d)=c^{d^{\delta}}$ for some $c>1$ and $\delta>0$, and functions $f(t) \not \equiv 1$ for which $f(x) f(y) \leq f(x+y)$ for all $x, y \in[1, \infty)$. 


\section{The proofs}

Here we present the proofs of the results from Section 2. To this end we only require elementary methods from analytic number theory and calculus. Using more sophisticated methods it might be possible to improve the involved error terms considerably. However, it is not the object of this paper to provide optimal error terms involved in the asymptotic relations.

First of all note that

$$
\begin{aligned}
\sum_{d \leq x} f(d)\left\{\frac{x}{d}\right\} & =x \sum_{d \leq x} \frac{f(d)}{d}-\sum_{n \leq x} f * \mathbf{1}(n) \\
& =x \sum_{d \leq x} \frac{f(d)}{d}-\sum_{n \leq x} \sum_{m \leq x / n} f(m),
\end{aligned}
$$

where $f * g$ is the convolution of two arithmetic functions $f$ and $g$ and $\mathbf{1}(d)=1$ for $d \in \mathbb{N}$.

In the following we will use Euler's summation formula (see, for example, [1] for a proof) which states that if a function $g(t)$ has a continuous derivative $g^{\prime}(t)$ on the interval $[1, x]$, then

$$
\sum_{d \leq x} g(d)=\int_{1}^{x} g(t) \mathrm{d} t+\int_{1}^{x}\{t\} g^{\prime}(t) \mathrm{d} t-g(x)\{x\}+g(1) .
$$

Lemma 1 Assume that the function $g(t)$ has a continuous derivative $g^{\prime}(t)$ on the interval $[1, x]$, then for $1 \leq y \leq x$ we have

$$
\sum_{n \leq y} g\left(\frac{x}{n}\right)=\int_{1}^{y} g\left(\frac{x}{t}\right) \mathrm{d} t-\int_{x / y}^{x}\left\{\frac{x}{t}\right\} g^{\prime}(t) \mathrm{d} t+g(x)-\{y\} g\left(\frac{x}{y}\right) .
$$

Proof. We have

$$
\begin{aligned}
\int_{x / y}^{x}\left\lfloor\frac{x}{t}\right\rfloor g^{\prime}(t) \mathrm{d} t & =\sum_{n=1}^{\lfloor y\rfloor-1} n \int_{x /(n+1)}^{x / n} g^{\prime}(t) \mathrm{d} t+\lfloor y\rfloor \int_{x / y}^{x /\lfloor y\rfloor} g^{\prime}(t) \mathrm{d} t \\
& =\sum_{n \leq y} g\left(\frac{x}{n}\right)-\lfloor y\rfloor g\left(\frac{x}{y}\right) .
\end{aligned}
$$

Integration by parts yields

$$
\int_{x / y}^{x} \frac{x}{t} g^{\prime}(t) \mathrm{d} t=g(x)-y g\left(\frac{x}{y}\right)+\int_{1}^{y} g\left(\frac{x}{t}\right) \mathrm{d} t .
$$

Hence

$$
\int_{x / y}^{x}\left\{\frac{x}{t}\right\} g^{\prime}(t) \mathrm{d} t=g(x)-\{y\} g\left(\frac{x}{y}\right)+\int_{1}^{y} g\left(\frac{x}{t}\right) \mathrm{d} t-\sum_{n \leq y} g\left(\frac{x}{n}\right)
$$

and the result follows. 
We can give the proof of Proposition 1.

Proof. From (9) for $f(t)=1_{A}(t)$ we obtain

$$
\sum_{d \leq x} 1_{A}(d)\left\{\frac{x}{d}\right\}=x \sum_{d \leq g^{-1}(x)} \frac{1}{g(d)}-\sum_{n \leq x} 1_{A} * \mathbf{1}(n) .
$$

Now we use Dirichlet's hyperbola method (see, for example, [1]) and obtain for $1 \leq y \leq x$,

$$
\begin{array}{rl}
\sum_{n \leq x} 1_{A} & * \mathbf{1}(n)=\sum_{n \leq y} \sum_{m \leq x / n} 1_{A}(m)+\sum_{m \leq x / y} 1_{A}(m)\left\lfloor\frac{x}{m}\right\rfloor-\lfloor y\rfloor \sum_{n \leq x / y} 1_{A}(n) \\
= & \sum_{n \leq y}\left\lfloor g^{-1}\left(\frac{x}{n}\right)\right\rfloor+\sum_{m \leq g^{-1}(x / y)}\left\lfloor\frac{x}{g(m)}\right\rfloor-\lfloor y\rfloor\left\lfloor g^{-1}\left(\frac{x}{y}\right)\right\rfloor \\
= & \sum_{n \leq y} g^{-1}\left(\frac{x}{n}\right)+\sum_{m \leq g^{-1}(x / y)} \frac{x}{g(m)}-y g^{-1}\left(\frac{x}{y}\right)+O\left(\max \left(y, g^{-1}\left(\frac{x}{y}\right)\right)\right) .
\end{array}
$$

Choose $y(x)=y \in[1, x]$ to be the unique solution of $y=g^{-1}(x / y)$. (Such a $y$ exists, since $g$ is continuous and increasing and hence also $g^{-1}$, and since $g^{-1}(x / x)=g^{-1}(1) \leq$ $x$ and $g^{-1}(x / 1) \geq 1$.) Then we obtain

$$
\sum_{n \leq x} 1_{A} * \mathbf{1}(n)=\sum_{n \leq y} g^{-1}\left(\frac{x}{n}\right)+\sum_{m \leq y} \frac{x}{g(m)}-y^{2}+O(y) .
$$

With Lemma 1 and with (11) we obtain

$$
\begin{aligned}
\sum_{n \leq x} 1_{A} * \mathbf{1}(n)= & \int_{1}^{y} g^{-1}\left(\frac{x}{t}\right) \mathrm{d} t-\int_{x / y}^{x}\left\{\frac{x}{t}\right\}\left(g^{-1}\right)^{\prime}(t) \mathrm{d} t+g^{-1}(x)-\{y\} g^{-1}\left(\frac{x}{y}\right) \\
& +x\left(\int_{1}^{g^{-1}(x / y)} \frac{\mathrm{d} t}{g(t)}+\int_{1}^{g^{-1}(x / y)}\{t\}\left(\frac{1}{g}\right)^{\prime}(t) \mathrm{d} t+\frac{1}{g(1)}-\frac{y}{x}\{y\}\right) \\
& -y^{2}+O(y) .
\end{aligned}
$$

Now

$$
\int_{1}^{g^{-1}(x / y)}\{t\}\left(\frac{1}{g}\right)^{\prime}(t) \mathrm{d} t=\int_{1}^{\infty}\{t\}\left(\frac{1}{g}\right)^{\prime}(t) \mathrm{d} t+O\left(\frac{y}{x}\right) .
$$

Using the substitution $z=g(t)$ and integration by parts we also obtain

$$
\begin{aligned}
\int_{1}^{g^{-1}(x / y)} \frac{\mathrm{d} t}{g(t)} & =\int_{g(1)}^{x / y} \frac{1}{z} \frac{\mathrm{d} z}{g^{\prime}\left(g^{-1}(z)\right)}=\int_{g(1)}^{x / y} \frac{1}{z}\left(g^{-1}\right)^{\prime}(z) \mathrm{d} z \\
& =\frac{g^{-1}(x / y)}{x / y}-\frac{1}{g(1)}+\int_{g(1)}^{x / y} \frac{g^{-1}(z)}{z^{2}} \mathrm{~d} z .
\end{aligned}
$$


Furthermore, using the substitution $z=x / t$ we obtain

$$
\int_{1}^{y} g^{-1}\left(\frac{x}{t}\right) \mathrm{d} t=x \int_{x / y}^{x} \frac{g^{-1}(z)}{z^{2}} \mathrm{~d} z
$$

With these formulas we get

$$
\begin{aligned}
\sum_{n \leq x} 1_{A} * \mathbf{1}(n)= & x \int_{g(1)}^{x} \frac{g^{-1}(z)}{z^{2}} \mathrm{~d} z-\int_{x / y}^{x}\left\{\frac{x}{t}\right\}\left(g^{-1}\right)^{\prime}(t) \mathrm{d} t+g^{-1}(x) \\
& +x \int_{1}^{\infty}\{t\}\left(\frac{1}{g}\right)^{\prime}(t) \mathrm{d} t+O(y) .
\end{aligned}
$$

We consider $x \sum_{d \leq g^{-1}(x)} 1 / g(d)$. From (11) we obtain

$$
x \sum_{d \leq g^{-1}(x)} \frac{1}{g(d)}=x\left(\int_{1}^{g^{-1}(x)} \frac{\mathrm{d} t}{g(t)}+\int_{1}^{g^{-1}(x)}\{t\}\left(\frac{1}{g}\right)^{\prime}(t) \mathrm{d} t+\frac{1}{g(1)}-\frac{\{x\}}{g(x)}\right) .
$$

As above we have

$$
\int_{1}^{g^{-1}(x)} \frac{\mathrm{d} t}{g(t)}=\frac{g^{-1}(x)}{x}-\frac{1}{g(1)}+\int_{g(1)}^{x} \frac{g^{-1}(z)}{z^{2}} \mathrm{~d} z
$$

and

$$
\int_{1}^{g^{-1}(x)}\{t\}\left(\frac{1}{g}\right)^{\prime}(t) \mathrm{d} t=\int_{1}^{\infty}\{t\}\left(\frac{1}{g}\right)^{\prime}(t) \mathrm{d} t+O(1 / x) .
$$

Therefore we obtain

$$
x \sum_{d \leq g^{-1}(x)} \frac{1}{g(d)}=g^{-1}(x)+x \int_{g(1)}^{x} \frac{g^{-1}(z)}{z^{2}} \mathrm{~d} z+x \int_{1}^{\infty}\{t\}\left(\frac{1}{g}\right)^{\prime}(t) \mathrm{d} t-\frac{x\{x\}}{g(x)}+O(1) .
$$

Inserting (14) and (13) into (12) gives

$$
\sum_{d \leq x} 1_{A}(d)\left\{\frac{x}{d}\right\}=\int_{x / y}^{x}\left\{\frac{x}{t}\right\}\left(g^{-1}\right)^{\prime}(t) \mathrm{d} t+O(y)=\int_{1}^{x}\left\{\frac{x}{t}\right\}\left(g^{-1}\right)^{\prime}(t) \mathrm{d} t+O(y)
$$

as desired.

We give the proof of Theorem 1 .

Proof. Assume that $g(t)=a_{r} t^{r}+a_{r-1} t^{r-1}+\ldots+a_{0}$ with $a_{i} \in \mathbb{N}_{0}$ for $0 \leq i \leq r$ and $a_{r} \neq 0$. First we show two properties of $\left(g^{-1}\right)^{\prime}$.

(i) Since

$$
\lim _{t \rightarrow \infty} \frac{g^{-1}(t)}{\left(t / a_{r}\right)^{1 / r}}=\lim _{y \rightarrow \infty} \frac{y}{\left(g(y) / a_{r}\right)^{1 / r}}=1
$$


it follows that for every $x>0$ we have

$$
\lim _{t \rightarrow \infty} \frac{g^{-1}(x t)}{g^{-1}(t)}=x^{1 / r}
$$

Now we have

$$
\begin{aligned}
\frac{\left(g^{-1}\right)^{\prime}(x t)}{\left(g^{-1}\right)^{\prime}(t)} & =\frac{g^{\prime}\left(g^{-1}(t)\right)}{g^{\prime}\left(g^{-1}(x t)\right)} \\
& =\frac{a_{r} r\left(g^{-1}(t)\right)^{r-1}+a_{r-1}(r-1)\left(g^{-1}(t)\right)^{r-2}+\ldots+a_{1}}{a_{r} r\left(g^{-1}(x t)\right)^{r-1}+a_{r-1}(r-1)\left(g^{-1}(x t)\right)^{r-2}+\ldots+a_{1}} \\
& =\frac{a_{r} r+O\left(1 / g^{-1}(t)\right)}{a_{r} r\left(\frac{g^{-1}(x t)}{g^{-1}(t)}\right)^{r-1}+a_{r-1}(r-1)\left(\frac{g^{-1}(x t)}{g^{-1}(t)}\right)^{r-2} \frac{1}{g^{-1}(t)}+\ldots+a_{1} \frac{1}{g^{-1}(t)}} .
\end{aligned}
$$

With $g(t)$ also $g^{-1}(t)$ tends to infinity as $t$ increases. Therefore and with (16) it follows that for every $x>0$ we have

$$
\lim _{t \rightarrow \infty} \frac{\left(g^{-1}\right)^{\prime}(x t)}{\left(g^{-1}\right)^{\prime}(t)}=x^{-1+1 / r}
$$

We remark that this property means that $\left(g^{-1}\right)^{\prime}$ is regularly varying ${ }^{1}$.

(ii) There exists an absolute constant $c_{1}>0$ such that

$$
\begin{aligned}
\frac{\left(g^{-1}\right)^{\prime}(x / t)}{\left(g^{-1}\right)^{\prime}(x)} & =\frac{g^{\prime}\left(g^{-1}(x)\right)}{g^{\prime}\left(g^{-1}(x / t)\right)} \\
& =\frac{a_{r} r\left(g^{-1}(x)\right)^{r-1}+\ldots+a_{1}}{a_{r} r\left(g^{-1}(x / t)\right)^{r-1}+\ldots+a_{1}} \\
& \leq c_{1}\left(\frac{g^{-1}(x)}{g^{-1}(x / t)}\right)^{r-1} \\
& =c_{1} t^{1-1 / r}\left(\frac{g^{-1}(x) /\left(x / a_{r}\right)^{1 / r}}{g^{-1}(x / t) /\left(x /\left(t a_{r}\right)\right)^{1 / r}}\right)^{r-1} .
\end{aligned}
$$

From (15) it follows on the one hand that $g^{-1}(x) /\left(x / a_{r}\right)^{1 / r} \leq 2$ for all $x$ large enough and on the other hand that there is a $y_{0}>0$ such that $g^{-1}(x / t) /\left(x /\left(t a_{r}\right)\right)^{1 / r} \geq 1 / 2$ whenever $x / t \geq y_{0}$. If $1 \leq x / t \leq y_{0}$ we have

$$
\frac{g^{-1}(x / t)}{\left(x /\left(t a_{r}\right)\right)^{1 / r}} \geq \min _{1 \leq y \leq y_{0}} \frac{g^{-1}(y)}{\left(y / a_{r}\right)^{1 / r}}>0 .
$$

\footnotetext{
${ }^{1}$ A continuous function $f(t)$ is said to be regularly varying, if $f(x t) / f(t)$ tends to some positive limit $h(x)$ as $t \rightarrow \infty$ for every $x>0$. It is known that in this case

a) $h(x)=x^{\alpha}$ for some finite $\alpha$ and that

b) the convergence is uniform on any interval $[a, b]$ with $0<a<b<\infty$.

The notion of regularly varying functions goes back to Karamata [2,3] (see also [8] for more information).
} 
Combining these considerations with (18) it follows that there exists a $c>0$ such that

$$
\frac{\left(g^{-1}\right)^{\prime}(x / t)}{\left(g^{-1}\right)^{\prime}(x)} \leq c t^{1-1 / r} \text { for all } 1 \leq t \leq x .
$$

Now we follow Mercier and Nowak [6, Proof of (9)] to show that

$$
\lim _{x \rightarrow \infty} \int_{1}^{x} h(x, t) t^{-1-1 / r} \mathrm{~d} t=0,
$$

where $h(x, t):=t^{-1+1 / r}\left(g^{-1}\right)^{\prime}(x / t) /\left(g^{-1}\right)^{\prime}(x)-1$. Let $\varepsilon>0$. According to (19) we have

$$
\left|\int_{a}^{x} h(x, t) t^{-1-1 / r} \mathrm{~d} t\right| \leq c \int_{a}^{x} t^{-1-1 / r} \mathrm{~d} t+\int_{a}^{x} t^{-1-1 / r} \mathrm{~d} t \leq \frac{c r}{a^{1 / r}}+\frac{1}{\alpha+1} \frac{1}{a^{1 / r}}<\frac{\varepsilon}{2}
$$

for $a$ large enough and arbitrary $x \geq a$. Fix such an $a$ and choose $x$ so large that $|h(x, t)|<$ $t^{-1+1 / r} \varepsilon / 2$ for $1 \leq t \leq a$. This is possible according to (17) and since the convergence of $\left(g^{-1}\right)^{\prime}(x / t) /\left(g^{-1}\right)^{\prime}(x)$ for $x \rightarrow \infty$ is uniform on the interval $1 / a \leq 1 / t \leq 1$. Then we have

$$
\left|\int_{1}^{a} h(x, t) t^{-1-1 / r} \mathrm{~d} t\right| \leq \frac{\varepsilon}{2} \int_{1}^{a} t^{-2} \mathrm{~d} t<\frac{\varepsilon}{2}
$$

Now (20) follows from (21) and (22).

Note that $\int_{1}^{x}\{x / t\}\left(g^{-1}\right)^{\prime}(t) \mathrm{d} t=x \int_{1}^{x}\{t\}\left(g^{-1}\right)^{\prime}(x / t) t^{-2} \mathrm{~d} t$. Hence (20) implies that

$$
\left|\frac{1}{x\left(g^{-1}\right)^{\prime}(x)} \int_{1}^{x}\left\{\frac{x}{t}\right\}\left(g^{-1}\right)^{\prime}(t) \mathrm{d} t-\int_{1}^{\infty}\{t\} t^{-1-1 / r} \mathrm{~d} t\right| \rightarrow 0 \quad \text { as } x \rightarrow \infty .
$$

Since $\int_{1}^{\infty}\{t\} t^{-1-1 / r} \mathrm{~d} t=c_{-1+1 / r}$ (see (5)) it follows that

$$
\int_{1}^{x}\left\{\frac{x}{t}\right\}\left(g^{-1}\right)^{\prime}(t) \mathrm{d} t \sim c_{-1+1 / r} x\left(g^{-1}\right)^{\prime}(x) \sim C_{-1+1 / r} g^{-1}(x) \quad \text { as } x \rightarrow \infty,
$$

where $C_{-1+1 / r}=\frac{1}{r} c_{-1+1 / r}$ is defined as in (7).

Let $y(x)=y \in[1, x]$ be the unique solution of $y=g^{-1}(x / y)$. Hence $y^{r+1} \leq y g(y)=x$ and therefore $y(x) \leq x^{1 /(r+1)}$. Since $g^{-1}(x) \sim\left(x / a_{r}\right)^{1 / r}$ for $x \rightarrow \infty$ it follows that

$$
\frac{y(x)}{g^{-1}(x)}=O\left(x^{-1 /(r(r+1))}\right)=o(1) \quad \text { as } x \rightarrow \infty .
$$

Since $\sum_{d \leq x} 1_{A}(d)=\left\lfloor g^{-1}(x)\right\rfloor$ the result follows from (23), (24) and Proposition 1.

For the proof of Proposition 2 we need the following lemma.

Lemma 2 If $f$ has a continuous derivative $f^{\prime}$ on the interval $[1, x]$, then

$$
\begin{aligned}
\sum_{d \leq x} f(d)\left\{\frac{x}{d}\right\}= & x \int_{1}^{x} \frac{\{t\}}{t^{2}} f\left(\frac{x}{t}\right) \mathrm{d} t-x \int_{1}^{x} \frac{\{t\}}{t^{2}} f(t) \mathrm{d} t+x \int_{1}^{x} \frac{\{t\}}{t^{2}}\left\{\frac{x}{t}\right\} f^{\prime}\left(\frac{x}{t}\right) \mathrm{d} t \\
& +\sum_{n \leq x} f\left(\frac{x}{n}\right)\left\{\frac{x}{n}\right\}+f(1)\{x\}-f(x)\{x\} .
\end{aligned}
$$


Proof. From Euler's summation formula (11) we obtain

$$
\sum_{d \leq x} \frac{f(d)}{d}=\int_{1}^{x} \frac{f(t)}{t} \mathrm{~d} t+\int_{1}^{x}\{t\} \frac{f^{\prime}(t) t-f(t)}{t^{2}} \mathrm{~d} t-\frac{f(x)}{x}\{x\}+f(1)
$$

and hence, using (10), we obtain

$$
\begin{aligned}
\sum_{d \leq x} f(d)\left\{\frac{x}{d}\right\}= & x\left(\int_{1}^{x} \frac{f(t)}{t} \mathrm{~d} t+\int_{1}^{x}\{t\} \frac{f^{\prime}(t) t-f(t)}{t^{2}} \mathrm{~d} t-\frac{f(x)}{x}\{x\}+f(1)\right) \\
& -\sum_{n \leq x}\left(\int_{1}^{x / n} f(t) \mathrm{d} t+\int_{1}^{x / n}\{t\} f^{\prime}(t) \mathrm{d} t-f\left(\frac{x}{n}\right)\left\{\frac{x}{n}\right\}+f(1)\right) \\
= & x \int_{1}^{x} \frac{f(t)}{t} \mathrm{~d} t-\sum_{n \leq x} \int_{1}^{x / n} f(t) \mathrm{d} t \\
& +x \int_{1}^{x}\{t\} \frac{f^{\prime}(t) t-f(t)}{t^{2}} \mathrm{~d} t-\sum_{n \leq x} \int_{1}^{x / n}\{t\} f^{\prime}(t) \mathrm{d} t \\
& +\sum_{n \leq x} f\left(\frac{x}{n}\right)\left\{\frac{x}{n}\right\}+f(1)\{x\}-f(x)\{x\}
\end{aligned}
$$

For $F(z):=\int_{1}^{x / z} f(t) \mathrm{d} t$ we have $F^{\prime}(z)=-x / z^{2} f(x / z)$ and hence with (11) we obtain

$$
\begin{aligned}
\sum_{n \leq x} \int_{1}^{x / n} f(t) \mathrm{d} t & =\int_{1}^{x} F(z) \mathrm{d} z+\int_{1}^{x}\{z\} F^{\prime}(z) \mathrm{d} z-F(x)\{x\}+F(1) \\
& =\int_{1}^{x} \int_{1}^{x / z} f(t) \mathrm{d} t \mathrm{~d} z-\int_{1}^{x}\{z\} \frac{x}{z^{2}} f\left(\frac{x}{z}\right) \mathrm{d} z+\int_{1}^{x} f(t) \mathrm{d} t \\
& =x \int_{1}^{x} \frac{f(t)}{t} \mathrm{~d} t-x \int_{1}^{x} \frac{\{t\}}{t^{2}} f\left(\frac{x}{t}\right) \mathrm{d} t
\end{aligned}
$$

Therefore we have

$$
x \int_{1}^{x} \frac{f(t)}{t} \mathrm{~d} t-\sum_{n \leq x} \int_{1}^{x / n} f(t) \mathrm{d} t=x \int_{1}^{x} \frac{\{t\}}{t^{2}} f\left(\frac{x}{t}\right) \mathrm{d} t
$$

Similarly, for $G(z):=\int_{1}^{x / z}\{t\} f^{\prime}(t) \mathrm{d} t$ we have $G^{\prime}(z)=-x / z^{2}\{x / z\} f^{\prime}(x / z)$ and hence with (11) it follows as above that

$$
\begin{aligned}
\sum_{n \leq x} \int_{1}^{x / n}\{t\} f^{\prime}(t) \mathrm{d} t & =\int_{1}^{x} G(z) \mathrm{d} z+\int_{1}^{x}\{z\} G^{\prime}(z) \mathrm{d} t-G(x)\{x\}+G(1) \\
& =x \int_{1}^{x}\{t\} \frac{f^{\prime}(t)}{t} \mathrm{~d} t-x \int_{1}^{x} \frac{\{t\}}{t^{2}}\left\{\frac{x}{t}\right\} f^{\prime}\left(\frac{x}{t}\right) \mathrm{d} t
\end{aligned}
$$


Therefore we have

$$
\begin{aligned}
x \int_{1}^{x}\{t\} \frac{f^{\prime}(t) t-f(t)}{t^{2}} \mathrm{~d} t-\sum_{n \leq x} \int_{1}^{x / n}\{t\} f^{\prime}(t) \mathrm{d} t \\
=-x \int_{1}^{x} \frac{\{t\}}{t^{2}} f(t) \mathrm{d} t+x \int_{1}^{x} \frac{\{t\}}{t^{2}}\left\{\frac{x}{t}\right\} f^{\prime}\left(\frac{x}{t}\right) \mathrm{d} t .
\end{aligned}
$$

Inserting (26) and (27) into (25) yields the desired result.

Furthermore, we will need some elementary and well-known asymptotic formulas whose proof can be found, for example, in [1]. We collect them in the following lemma.

Lemma 3 For $x \geq 1$ we have:

(i) $\sum_{n \leq x} 1 / n^{s}=x^{1-s} /(1-s)+\zeta(s)+O\left(x^{-s}\right)$ for $s>0, s \neq 1$.

(ii) $\sum_{n \leq x} n^{\alpha}=\frac{x^{\alpha+1}}{\alpha+1}+O\left(x^{\alpha}\right)$ for $\alpha \geq 0$.

(iii) $\sum_{n \leq x} 1 / n=\log x+\gamma+O(1 / x)$.

Let $\sigma_{\alpha}(n):=\sum_{d \mid n} d^{\alpha}$ be the sum of $\alpha$-th powers of the divisors of $n$. Note that $\sigma_{\alpha}(n)=$ $f * \mathbf{1}(n)$ where $f(d)=d^{\alpha}$. We have the following asymptotic relations.

Lemma 4 For $x \geq 1$ we have:

(i) $\sum_{n \leq x} \sigma_{\alpha}(n)=\frac{\zeta(\alpha+1)}{\alpha+1} x^{\alpha+1}+O\left(x^{\alpha}\right)$ for $\alpha>1$.

(ii) $\sum_{n \leq x} \sigma_{1}(n)=\frac{\zeta(2)}{2} x^{2}+O\left(x(\log x)^{2 / 3}\right)$.

(iii) $\sum_{n \leq x} \sigma_{\alpha}(n)=\frac{\zeta(\alpha+1)}{\alpha+1} x^{\alpha+1}+\zeta(1-\alpha) x+O\left(x^{(\alpha+1) / 2}\right)$ for $0<\alpha<1$.

(iv) $\sum_{n \leq x} \sigma_{0}(n)=x \log x+(2 \gamma-1) x+O(\sqrt{x})$.

The results from (i) and (iv) are well-known; see, for example, [1]. A proof for (ii) can be found in [11]. The result from (iii) is an improvement of [1, Theorem 3.5] for $0<\alpha<1$. We found the result nowhere in literature but it can easily be shown by using Dirichlet's hyperbola method.

We give the proof of Proposition 2.

Proof. For $\alpha \geq 0$ we obtain from (9) that

$$
\sum_{d \leq x} d^{\alpha}\left\{\frac{x}{d}\right\}=x \sum_{d \leq x} d^{\alpha-1}-\sum_{n \leq x} \sigma_{\alpha}(n)
$$

and the result follows from Lemma 3 and 4. 
For $\alpha<0$ it follows from Lemma 2 that

$$
\sum_{d \leq x} d^{\alpha}\left\{\frac{x}{d}\right\}=x^{1+\alpha} \int_{1}^{x} \frac{\{t\}}{t^{2+\alpha}} \mathrm{d} t-x \int_{1}^{x} \frac{\{t\}}{t^{2-\alpha}} \mathrm{d} t+x^{\alpha} \sum_{d \leq x} d^{-\alpha}\left\{\frac{x}{d}\right\}+O(1) .
$$

We have

$$
\begin{aligned}
\int_{1}^{x} \frac{\{t\}}{t^{2-\alpha}} \mathrm{d} t & =\int_{1}^{x} \frac{\mathrm{d} t}{t^{1-\alpha}}-\sum_{k=1}^{\lfloor x\rfloor} k \int_{k}^{k+1} \frac{\mathrm{d} t}{t^{2-\alpha}}-\lfloor x\rfloor \int_{\lfloor x\rfloor}^{x} \frac{\mathrm{d} t}{t^{2-\alpha}} \\
& =-\frac{1}{\alpha}-\frac{\zeta(1-\alpha)}{1-\alpha}+O\left(\frac{1}{x^{1-\alpha}}\right) .
\end{aligned}
$$

For the evaluation of $\int_{1}^{x}\{t\} t^{-2-\alpha} \mathrm{d} t$ we consider two cases:

- If $\alpha=-1$, then we have

$$
\begin{aligned}
\int_{1}^{x} \frac{\{t\}}{t} \mathrm{~d} t & =x-1-\int_{1}^{x} \frac{\lfloor t\rfloor}{t} \mathrm{~d} t \\
& =x-1-\sum_{k=1}^{x-1} k \int_{k}^{k+1} \frac{\mathrm{d} t}{t}-\lfloor x\rfloor \int_{\lfloor x\rfloor}^{x} \frac{\mathrm{d} t}{t} \\
& =x-\sum_{k \leq x} k \log (1+1 / k)+O(1) \\
& =\frac{1}{2} \sum_{k \leq x} \frac{1}{k}+O(1)=\frac{1}{2} \log x+O(1)
\end{aligned}
$$

where we used that $\log (1+y)=y-\frac{1}{2} y^{2}+O\left(y^{3}\right)$. Hence

$$
\begin{aligned}
\sum_{d \leq x} \frac{1}{d}\left\{\frac{x}{d}\right\} & =\int_{1}^{x} \frac{\{t\}}{t} \mathrm{~d} t-x \int_{1}^{x} \frac{\{t\}}{t^{3}} \mathrm{~d} t+\frac{1}{x} \sum_{d \leq x} d\left\{\frac{x}{d}\right\}+O(1) \\
& =\frac{1}{2} \log x+O\left((\log x)^{2 / 3}\right) .
\end{aligned}
$$

- If $-1<\alpha<0$, then it follows in the same way as above that

$$
\int_{1}^{x} \frac{\{t\}}{t^{2+\alpha}} \mathrm{d} t=\frac{1}{\alpha}-\frac{\zeta(1+\alpha)}{1+\alpha}+O\left(\frac{1}{x^{1+\alpha}}\right)
$$

and hence

$$
\sum_{d \leq x} d^{\alpha}\left\{\frac{x}{d}\right\}=x^{\alpha+1}\left(\frac{1}{\alpha}-\frac{\zeta(\alpha+1)}{\alpha+1}\right)+O\left(x^{(\alpha+1) / 2}\right) .
$$

We give the proof of Theorem 2 .

Proof. For $\alpha \geq-1$ the result follows from Proposition 2 and for $\alpha<-1$ the result follows from the fact that an asymptotic relation of the form (8) can only hold true for some $f(t)$ if $\sum_{d=1}^{\infty} f(d)=\infty$. 


\section{Acknowledgement}

The author thanks Gerhard Larcher and Stefan Steinerberger for valuable discussions and suggestions.

\section{References}

[1] Apostol, T.M.: Introduction to Analytic Number Theory. Springer-Verlag, New York-Heidelberg-Berlin 1976.

[2] Karamata, J.: Sur un mode de croissance régulière des functions. Mathematica 4 (1930), 38-53.

[3] Karamata, J.: Sur un mode de croissance régulière. Théorèmes fondamentaux. Bull. Soc. Math. France 61 (1933), 55-62.

[4] Mercier, A.: Comportement asymptotique de $\sum_{n \leq x} n^{a}\{f(x / n)\}$. Ann. Sci. Math. Québec 9 (1985), 199202.

[5] Mercier, A.: Sums containing the fractional part of numbers. Rocky Mountain J. Math. 15 (1985), 513520.

[6] Mercier, A.; Nowak, W.G.: On the asymptotic behaviour of sums $\sum g(n)\{x / n\}^{k}$. Monatsh. Math. 99 (1985), 213-221.

[7] Pillichshammer, F.: Euler's constant and averages of fractional parts. Amer. Math. Monthly 117 (2010), 78-83.

[8] Seneta, E.: Regularly varying functions. Lecture Notes in Mathematics, Vol. 508. Springer-Verlag, BerlinNew York 1976.

[9] Smith, R.A.; Subbarao, M.V.: The average number of divisors in arithmetic progression. Canad. Math. Bull. 24 (1981), 37-41.

[10] de la Vallée Poussin, C.: Sur les valeurs moyennes de certaines fonctions arithmétiques. Annales de la Société Scientifique de Bruxelles 22 (1898), 84-90.

[11] Walfisz, A.: Exponentialsummen in der neueren Zahlentheorie. Mathematische Forschungsberichte, XV. VEB Deutscher Verlag der Wissenschaften, Berlin 1963.

Friedrich Pillichshammer

Institut für Finanzmathematik

Universität Linz

Altenbergerstraße 69

A-4040 Linz, Austria

e-mail: friedrich.pillichshammer@jku.at 